\title{
CMOS-compatible LVOF-based visible microspectrometer
}

\author{
Arvin Emadi*, Huaiwen Wu, Ger de Graaf and Reinoud F. Wolffenbuttel \\ Delft University of Technology, Faculty of EEMCS, Department of ME/EI, Mekelweg 4, \\ 2628 CD Delft, The Netherlands
}

\begin{abstract}
This paper reports on a CMOS-Compatible Linear Variable Optical Filter (LVOF) visible micro-spectrometer. The CMOS-compatible post process for fabrication of the LVOF has been used for integration of the LVOF with a CMOS chip containing a 128-element photodiode array and readout circuitry. Fabrication of LVOF involves a process for fabrication of very small taper angles, ranging from $0.001^{\circ}$ to $0.1^{\circ}$, in $\mathrm{SiO}_{2}$. These layers can be fabricated flexibly in a resist layer by just one lithography step and a subsequent reflow process. The 3D pattern of the resist structures is subsequently transferred into $\mathrm{SiO}_{2}$ by appropriate etching. Complete LVOF fabrication involves CMOS-compatible deposition of a lower dielectric mirror using a stack of dielectrics on the wafer, tapered layer formation and deposition of the top dielectric mirror. The LVOF has been optimized for $580 \mathrm{~nm}-720 \mathrm{~nm}$ spectral operating range and has also been mounted on a CCD camera for characterization. The design of LVOF micro-spectrometer, the fabrication and characterization results are presented.
\end{abstract}

Keywords: Microspectrometer, Linear Variable Filter, CMOS-compatible optical sensors, tapered Fabry-Perot

\section{INTRODUCTION}

Single-chip optical micro-spectrometers have huge potential in many applications, such as identification of biomolecules, gas detection and in chemical analysis, because of their properties such as low-cost and low sample volume [1]-[5]. A small Linear Variable Optical Filter (LVOF) integrated with an array of optical detectors is a very suitable candidate for a micro-spectrometer that should feature both low unit cost and high resolving power [6]-[7]. Although grating based micro-spectrometers generally outperform optical resonance based systems, such as the Fabry-Perot etalon or the LVOF-based micro-spectrometer, in case of operation over a wide spectral bandwidth, LVOF-based microspectrometers are more suitable for operation with high resolving power over a narrow spectral band, as is required in spectral analysis around an absorption line [8]. IC-Compatible fabrication enables the fabrication of LVOFs as a postprocess in CMOS. Having the detector array and electronic circuits realized in CMOS prior to application of the postprocess offers opportunities for low unit costs in case of a high production volume.

The LVOF is basically a one-dimensional array of many Fabry-Perot (FP)-type of optical resonators. Rather than a huge number of discrete devices [9], the LVOF has a center layer (the resonator cavity) in the shape of a strip and a thickness that changes over its length. Dielectric mirrors are on either side. The spectral resolution of a Fabry-Perot interferometer is determined by surface flatness, parallelism between the two mirror surfaces and mirror reflectivity. The possibility to have high number of spectral channels in a LVOF spectrometer theoretically makes it possible to have spectral resolution better than $0.2 \mathrm{~nm}$ in the visible spectrum range using signal processing techniques. For a Fabry-Perot type of LVOF, the thickness variation of the cavity layer has to be in order of quarter of the wavelength and very well controlled, which makes fabrication of miniature LVOFs a technological challenge. The theoretical limit for the spectral resolving power of the LVOF-based spectrometer is the spectral bandwidth divided by the number of channels in the detector array. However, this is difficult to achieve when considering the Signal to Noise Ratio. This simple geometric optimum is only approached in case of a high SNR.

LVOF fabrication is based on reflow of a specially patterned layer of resist. Figure 1 shows the process steps for the fabrication of an IC-Compatible LVOF. The process starts with deposition of the lower dielectric mirror stack and the oxide layer that results in the cavity layer. Photoresist is spin coated as the next step and lithography is applied to define the strip-like structure in the resist layer to be reflown. A series of trenches of constant width and with variable spatial frequency or trenches of variable width and constant pitch are etched over the length of the strip of resist to vary the effective amount of resist per unit area. The subsequent reflow transfers this gradient volume of resist into a smooth tapered resist layer. The topography of the tapered resist layer is transformed into the thick oxide cavity layer by an appropriate plasma etching process. The process is completed by deposition of the top dielectric mirror stack. This first

Next-Generation Spectroscopic Technologies III, edited by Mark A. Druy, Christopher D. Brown, Richard A. Crocombe, Proc. of SPIE Vol. 7680, 76800W - @ 2010 SPIE - CCC code: 0277-786X/10/\$18 - doi: 10.1117/12.849844 
prototype of the LVOF is fabricated on a glass substrate. Fabrication directly on a CMOS detector chip as a compatible post-process is well possible and is also demonstrated on small die CMOS chips.

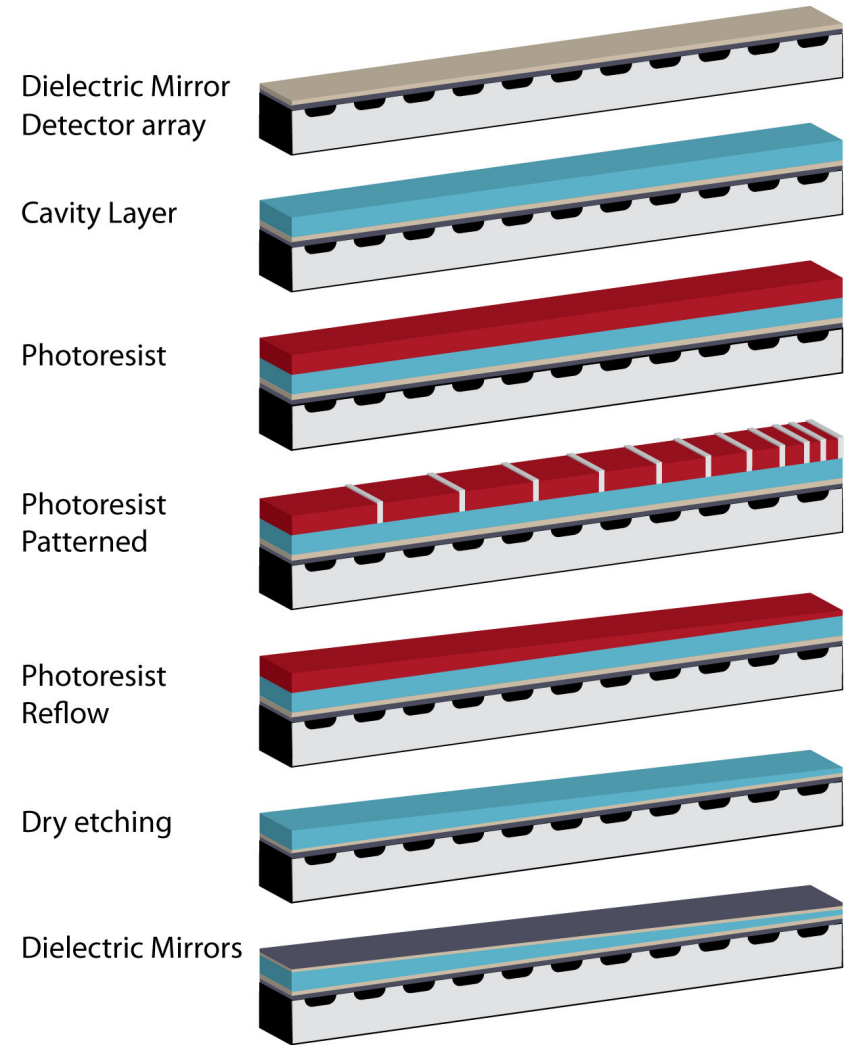

Figure 1. Process flow for fabrication of LVOF.

The LVOF design presented here is based on operation in the wavelength range between $570 \mathrm{~nm}$ to $720 \mathrm{~nm}$. Spectrometers in this wavelength range are suitable for applications such as fluorescence spectroscopy of plants and $\mathrm{H}-\alpha$ spectroscopy. The performance characterization of the LVOF and related micro-spectrometer in this wavelength range can be tested using a Neon lamp, which has most of its major peaks in this wavelength range. The same principle of design can be applied to other wavelength ranges in visible, IR and UV. The difference would be in the choice of dielectric materials for the LVOF filter and suitable detector array. For near-infra red region, for example PolySi and $\mathrm{SiO}_{2}$ are to be used as dielectric materials [10] and MEMS fabricated thermopile arrays as detectors [11].

The dielectric multilayered Fabry-Perot LVOF consists of 15 alternative layers of $\mathrm{TiO}_{2}$ and $\mathrm{SiO}_{2}$. Table 1 shows the layers thicknesses required for a visible LVOF to cover the 570 to $720 \mathrm{~nm}$ spectral range based on optical simulations. Figure 2 shows the simulated spectral response for different thicknesses of the cavity layer, which gives a prediction of the spectral response of the LVOF at different positions along its length. As the table shows, the cavity layers thickness should vary between $800 \mathrm{~nm}$ and $1050 \mathrm{~nm}$ to cover the desired wavelength range. The simulation has been done with $\mathrm{TFCalc}^{\circledR} 3.3$ software [12]. The spectral bandwidth of the filter depends on reflection spectrum of dielectric mirrors and the order of the Fabry-Perot. Increasing the order of the cavity results in higher resolving power, but limits the operating free spectral range of Fabry-Perot etalon. The LVOF based on the Fabry-Perot etalon listed in Table 1 gives a half-power spectral bandwidth HPBW $=2.2 \mathrm{~nm}$ over $150 \mathrm{~nm}$ wavelength range. The HPBW of a LVOF is slightly larger than that of a corresponding Fabry-Perot, because of multiple reflections between the non-parallel mirrors [13]. 
Table 1. Thickness of the layers for visible LVOF

\begin{tabular}{|c|c|}
\hline Material & Thickness (nm) \\
\hline $\mathrm{TiO}_{2}$ & 60 \\
\hline $\mathrm{SiO}_{2}$ & 102 \\
\hline $\mathrm{TiO}_{2}$ & 60 \\
\hline $\mathrm{SiO}_{2}$ & 102 \\
\hline $\mathrm{TiO}_{2}$ & 60 \\
\hline $\mathrm{SiO}_{2}$ & 102 \\
\hline $\mathrm{TiO}_{2}$ & 60 \\
\hline $\mathrm{SiO}_{2}$ & $800-1050$ \\
\hline $\mathrm{TiO}_{2}$ & 60 \\
\hline $\mathrm{SiO}_{2}$ & 102 \\
\hline $\mathrm{TiO}_{2}$ & 60 \\
\hline $\mathrm{SiO}_{2}$ & 102 \\
\hline $\mathrm{TiO}_{2}$ & 60 \\
\hline $\mathrm{SiO}_{2}$ & 102 \\
\hline $\mathrm{TiO}_{2}$ & 60 \\
\hline
\end{tabular}

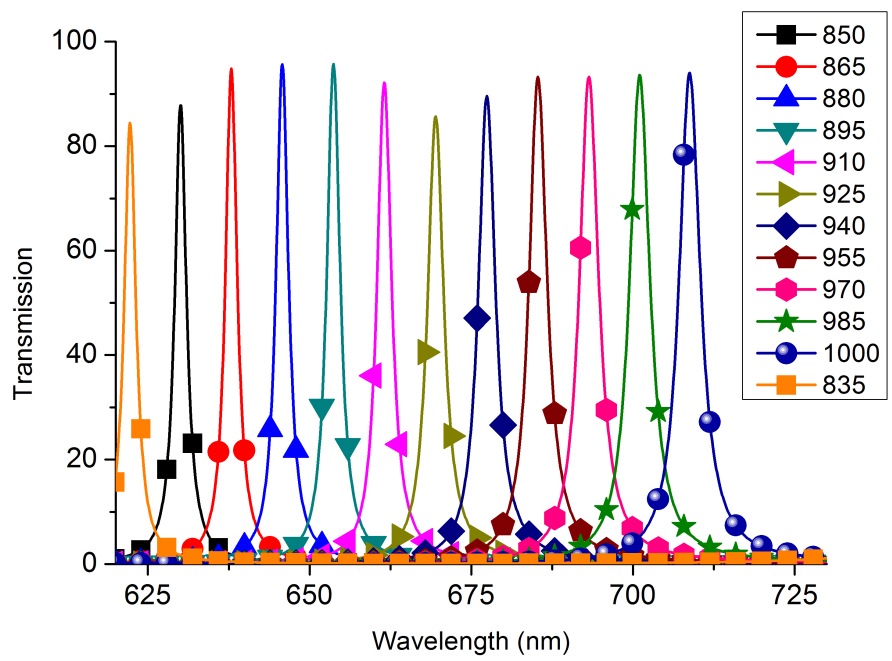

Figure 2. simulated spectral response of multi-layered Fabry-Perot for different cavity layer thicknesses, thickness values in nm.

Based on the simulation results it was decided to design for 2 types of LVOFs; the first with a length of $2.5 \mathrm{~mm}$ and the second with a length of $5 \mathrm{~mm}$. In the $5 \mathrm{~mm}$ long LVOF the thickness variation (slope) would be equal to $150 \mathrm{~nm} / 5 \mathrm{~mm}$. In case collimated light of a single wavelength is used to illuminate the strip, the light over a small length is transmitted, due to filtering property of the LVOF. The length of the transmitted light spot can be calculated as: $\Delta x=\frac{H P B W}{\theta}$, where $\theta$ denotes the slope of the LVOF. Using the values of HPBW=2.2 $\mathrm{nm}$ and above mentioned slope angle results in $\Delta x \approx 75 \mu \mathrm{m}$. This means for each single wavelength in the spectrum a region of $75 \mu \mathrm{m}$ in length is illuminated on the detector. Therefore, if a typical detector with $5 \mu \mathrm{m}$ pitch is used, about 15 pixels on the detector are illuminated. The structure of a LVOF based micro-spectrometer is shown in Figure 3. Light passes an aperture and collimating optics before being projected onto the LVOF, which is placed or deposited on the top of the detector. The entrance aperture in 
figure 3 can be larger than that typically used in grating-based micro-spectrometers, allowing more light entering the optical system. Consequently, the resolution of the micro-spectrometer depends primarily on the Half-Power BandWidth (HPBW) of the LVOF, rather than on the aperture size. To determine the size of aperture and focal length of the collimating lens the following equations can be used:

$d=\frac{D \cdot \varphi}{N A}$ and $f=\frac{D}{2 N A}$, in which $d$ is the diameter of the aperture, $D$ is size of the LVOF, $f$ is the focal length of the lens, $N A$ is entrance numerical aperture and $\varphi$ is maximum acceptable angle of incidence on the LVOF. For spectral resolution better than $2 \mathrm{~nm}, \varphi=5^{\circ}$ is an acceptable choice.

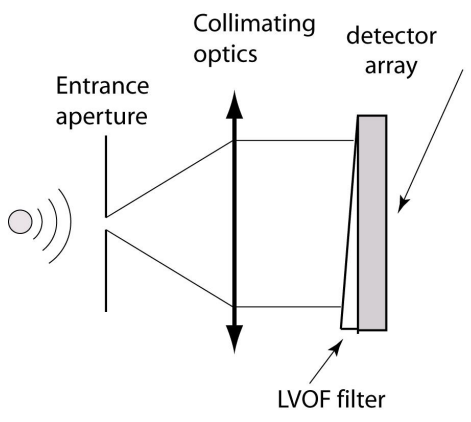

Figure 3. Structure of LVOF micro-spectrometer

The first fabrication step of the LVOF is the deposition dielectric mirrors, layers 1 to 7 , and a thick oxide of $1050 \mathrm{~nm}$, layer 8 , on a 4 inch glass wafer. The thick oxide layer is tapered in subsequent process steps. A FHR MS 150 sputter machine has been used for deposition of $\mathrm{TiO}_{2}$ and $\mathrm{SiO}_{2}$ films. $\mathrm{TiO}_{2}$ is deposited by reactive $\mathrm{DC}$ sputtering and $\mathrm{SiO}_{2}$ by reactive RF sputtering. The films can be deposited subsequently without breaking the vacuum. The films are optically characterized by ellipsometry and the data were used to refine the values used for the designed thicknesses. The thickness variation over the 4 inch wafer size is measured to be less than $2 \%$ for both films. The effect of such a relative thickness is shown in the simulation of reflectance of the optical filter, as shown in Figure 4. Thickness variations can cause $\pm 6 \mathrm{~nm}$ wavelength shift of the peak. This implies that fabricating a Fabry-Perot at an exact desired peak can be challenging. For a LVOF type of Fabry-Perot device this problem is resolved, since the sloped cavity layer makes it possible to cover the whole spectrum range of interest despite of wavelength shift. The 4 inch glass wafer is diced into 2 $\times 2 \mathrm{~cm}^{2}$ pieces prior to subsequent processing. 


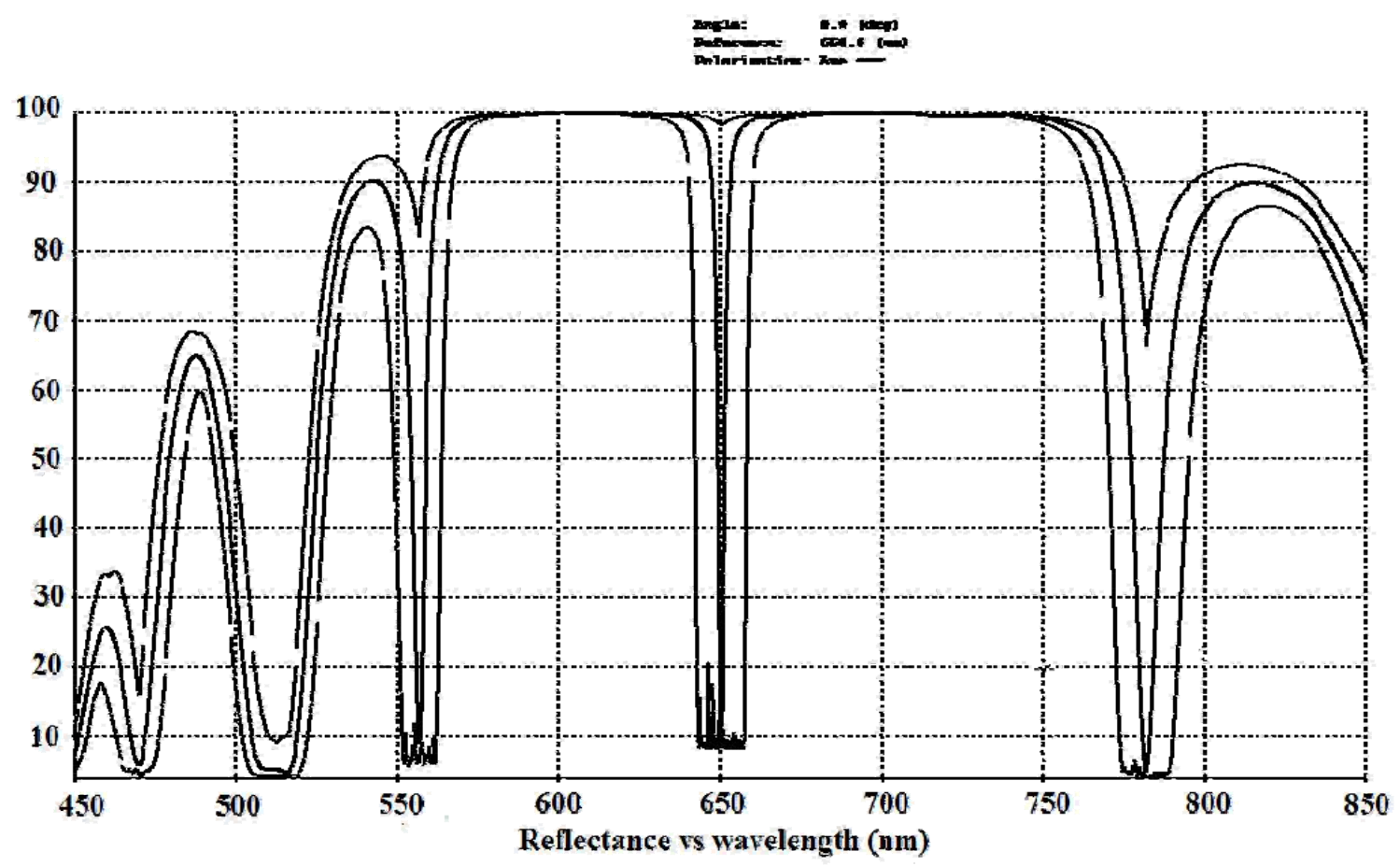

Figure 4. Simulated reflectance spectrum of multi-layered Fabry-Perot for $850 \mathrm{~nm}$ thickness of the cavity layer considering thickness variations in the films.

The $\mathrm{SiO}_{2}$ cavity layer is tapered in an IC-Compatible fabrication process. Initially, a tapered photoresist layer is formed by one step lithography and reflow of the resist. Subsequently, the topography of the tapered resist layer is transferred into $\mathrm{SiO}_{2}$ by RIE. With a single lithography step a pattern of trenches (or holes) is developed in a photoresist layer. The trench or holes size is $2 \mu \mathrm{m}$, which is the minimum possible with the available lithography. The trench density defines the local amount of material removed and is followed by reflow of this patterned photoresist to locally planarize the remaining strips of material. The result is an effective reduction of the resist layer thickness by a value defined by localized trench density. Hence a taper can be flexibly programmed by mask design to be from $0.001^{\circ}$ to $0.1^{\circ}$. This enables simultaneous fabrication of tapered layers of different angle. The mask is designed based on a geometrical model and FEM simulations using COMSOL [14]. Figure 5 shows the mask and one patterned photoresist structure before reflow. The mask contains patterns that will result in hill-shaped tapered resist layers of different angles after reflow.

The viscosity of the resist decreases rapidly at temperatures above the glass transition temperature and the material flows because of surface tension, while remaining coherent. Although the glass transition temperature of resist is relatively low value $\left(110-120^{\circ} \mathrm{C}\right)$, the resist does not flow well over the substrate due to its high viscosity. Another approach to decrease the viscosity of the resist is to expose it to its solvent vapor. The samples with patterned resist on the dielectric stack are exposed to PGMEA solvent vapor heated to $50^{\circ} \mathrm{C}$ for 3 hours and heated on hotplate at $110{ }^{\circ} \mathrm{C}$ for $10 \mathrm{~min}$. 


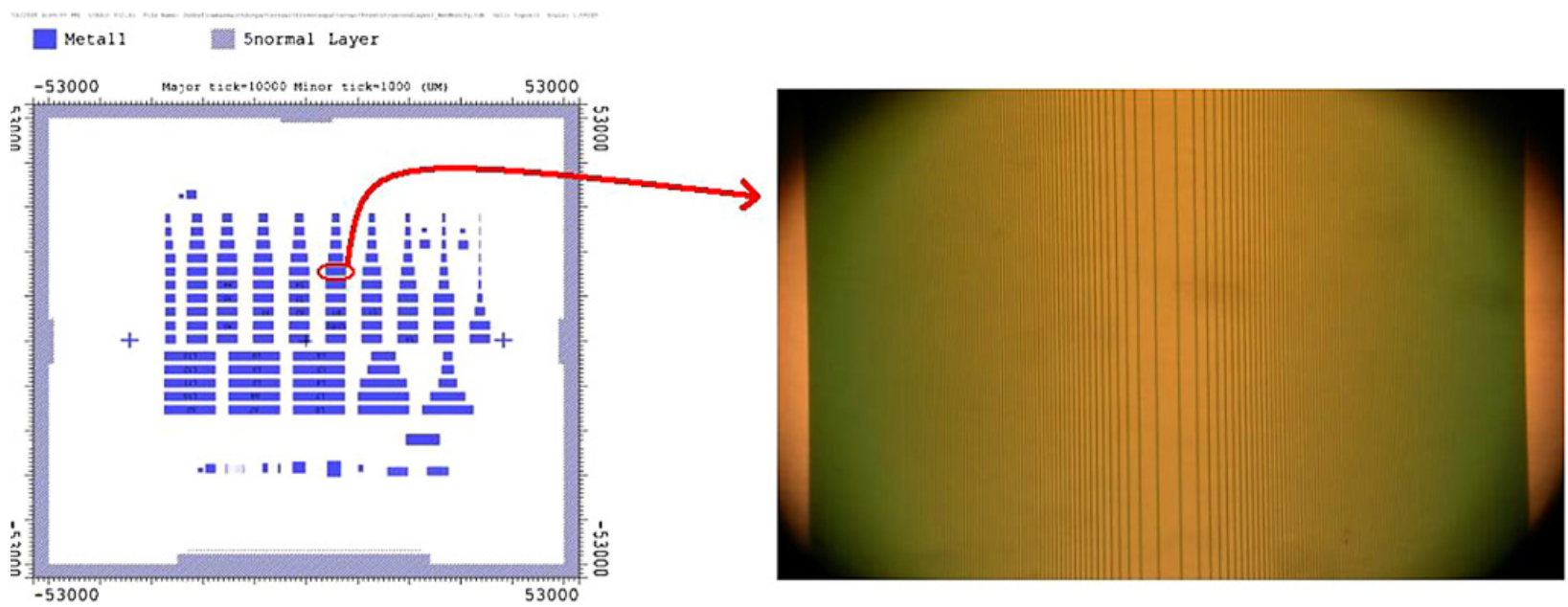

Figure 5. Left) Photo-mask for lithography. Right) patterned photoresist before reflow, trench density increase from the center toward the edges.

The process for transferring the resist structure into $\mathrm{SiO}_{2}$ is optimized to get an optically smooth surface. The process uses a mixture of $\mathrm{NF}_{3} 5 \mathrm{sccm}$, Ar $50 \mathrm{sccm}$ and $\mathrm{O}_{2} 5 \mathrm{sccm}$ at 2 mTorr with 100 Watt. The low chamber pressure will cause the etching process to be slow [15] and result in an acceptable surface roughness of $5 \mathrm{~nm}$ on the oxide after etching. The etch rate of the photoresist is $75 \mathrm{~nm} / \mathrm{min}$ while the etch rate of the $\mathrm{SiO}_{2}$ is $15 \mathrm{~nm} / \mathrm{min}$, which gives a resist $/ \mathrm{SiO}_{2}$ etch ratio of 5. A laser illuminates the thickest part of the resist structure during etching and the reflection from the surface is measured. Due to interference between resist, oxide layer and substrate, this will show exactly how much of the resist and $\mathrm{SiO}_{2}$ has been etched. The etching process is stopped after resist is removed from the thickest part and $50 \mathrm{~nm}$ of the $\mathrm{SiO}_{2}$ under it, is etched. After etching the samples are cleaned in acetone and further by Oxygen plasma to remove any remaining of photoresist. Subsequently, the dielectric stacks, layers 9 to 15 from table 1 , are deposited on the samples to complete the LVOFs. Figure 6 shows the 3D image of one completed LVOF using optical profilometer.

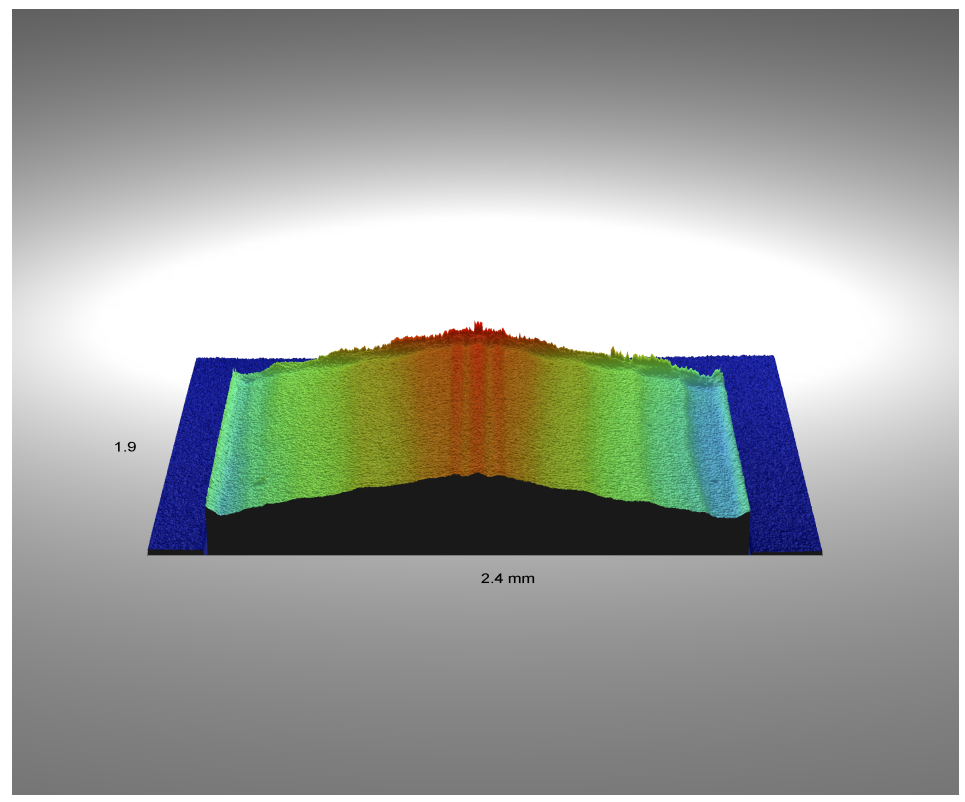

Figure 6. 3D profile of the completed hill-shaped LVOF. 


\section{INTEGRATION OF LVOF AND DETECTOR}

The LVOF is mounted on the top of a CMOS camera. A special C-mount lens holder has been fabricated to keep a lens and pinhole. Figure 7 shows LVOF on a glass substrate mounted on a CCD chip and the C-mount covering them.
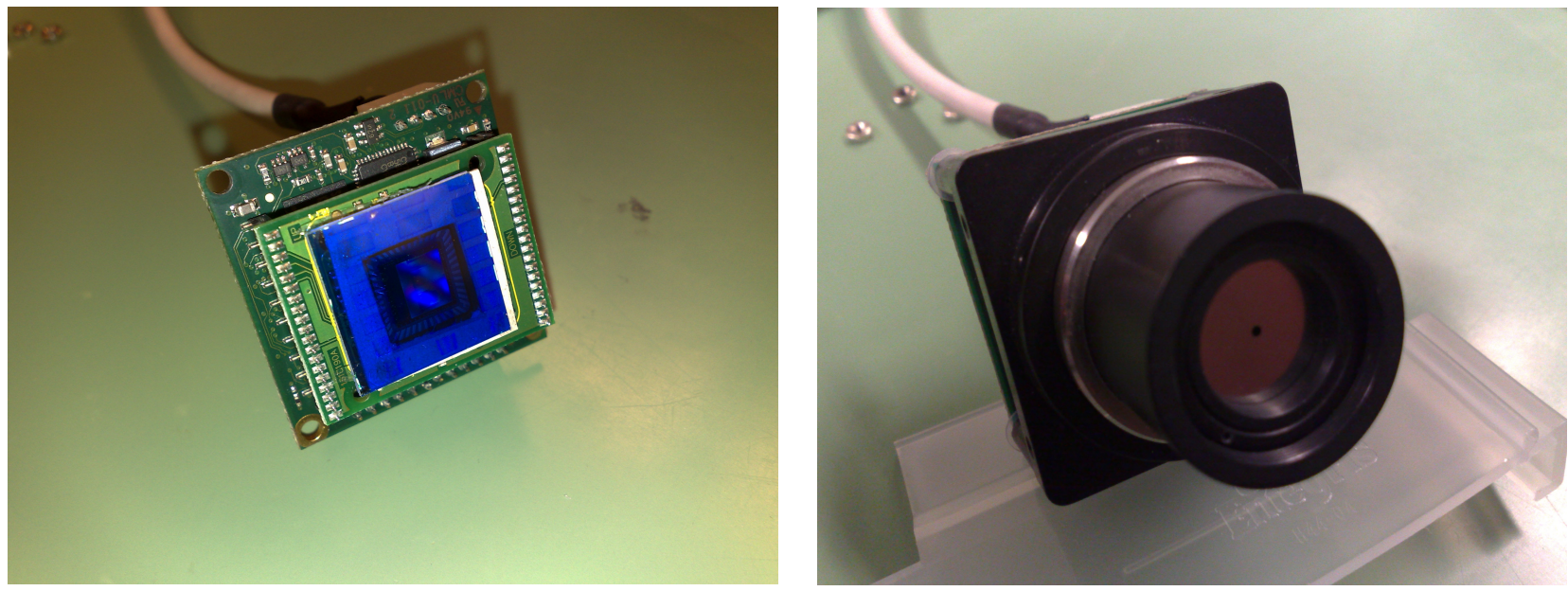

Figure 7. Left) LVOF on glass substrate on the CMOS detector. Right) C-mount lens-pinhole holder on the LVOF and CMOS camera to make the LVOF spectrometer.

The LVOF spectrometer is illuminated with light from a Neon lamp. Figure 8 shows the recorded image of the CMOS camera for two LVOFs, each with a different slope. The LVOF image on the left has $250 \mathrm{~nm}$ thickness slope and covers the entire spectral band of interest, while the LVOF on the right has $150 \mathrm{~nm}$ thickness slope and therefore does not cover the entire band. The recorded pattern is the result of spectrum of Neon lamp having few major peaks in $580 \mathrm{~nm}-720$ $\mathrm{nm}$ spectral range. Extracting the spectrum from the captured image requires calibration of the LVOF spectrometer and signal processing.
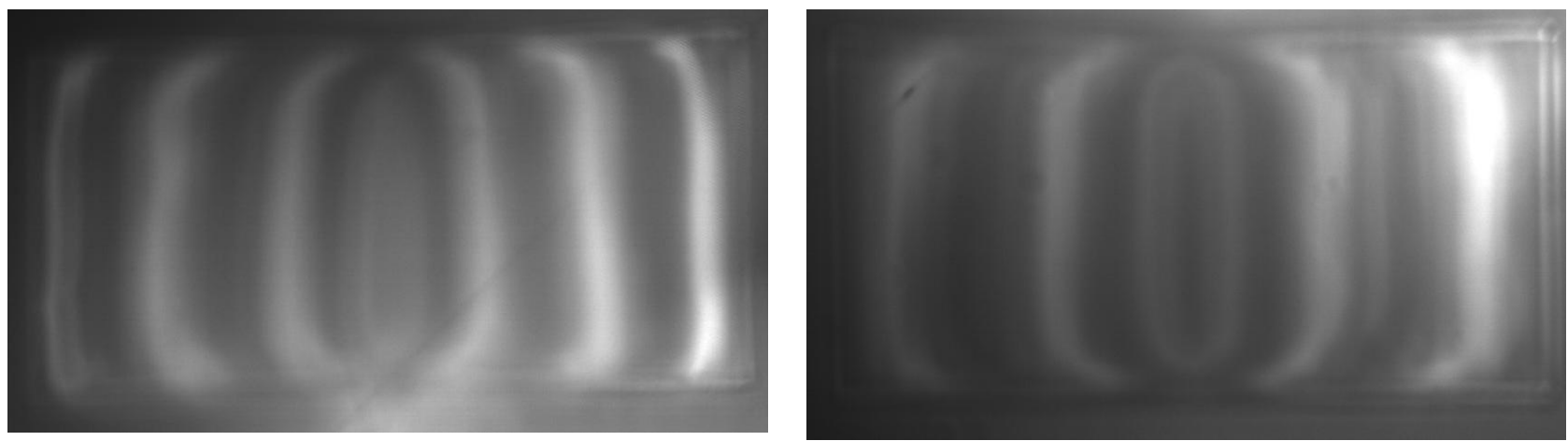

Figure 8. Recorded image on the CMOS camera when illuminated with Neon lamp.

Integration of the LVOF with a CMOS chip at the die-level and calibration of the LVOF spectrometer for actual spectral measurements are the subjects of future research. 


\section{ACKNOWLEDGMENT}

This work has been supported in part by the Dutch technology Foundation STW under grant DET.6667. Part of this work has been done at the Nanofabrication Laboratory of MC2, Chalmers University of Technology, through MC2ACCESS programme.

\section{REFERENCES}

[1] G. Minas, R. F. Wolffenbuttel and J. H. Correia. An array of highly selective Fabry-Perot optical channels for biological fluid analysis by optical absorption using a white light source for illumination, Journal of Optics A: Pure and Applied Optics, IOP Publisher,8, pp.272-278.

[2] J.C. Ribeiro, G. Minas, P. Turmezei, R.F.Wolffenbuttel and J.H. Correia, "A SU-8 fluidic microsystem for biological fluids analysis", Sensors and Actuators A123-124 (2005) pp. 77-81.

[3] G. Minas, R.F.Wolffenbuttel and J.H. Correia, "A lab-on-a-chip for spectrophotometric analysis of biological fluids", RSC Lab Chip, Vol. 5 No. 11, pp. 1303-1309.

[4] S. Grabarnik, R.F. Wolffenbuttel, A. Emadi, M. Loktev, E. Sokolova and G. Vdovin, "Planar double-grating microspectrometer", Optics Express, March 19 2007, Vol. 15, No. 6, pp. 3581-3588.

[5] L. Fonseca, R. Rubio, J. Santander, C. Calaza, N. Sabate, P. Ivanov, E. Figueras, I. Gracia, C. Cane, S. Udina, M. Moreno, S. Marco, "Qualitative and quantitative substance discrimination using a CMOS compatible non-specific NDIR microarray", Sensors and Actuators B: Chemical, Volume 141, Issue 2, 7 September 2009

[6] R. McLeod, T. Honda. Improving the spectral resolution of wedged etalons and linear variable filters with incidence angle, Optics Letters, Vol. 30, No. 19 (2005), pp 2647-2649.

[7] S.F. Pellicori, US Patent 4957371 - Wedge-filter spectrometer

[8] S. Grabarnik, Optical microspectrometers using imaging diffraction gratings, PhD thesis, Delft University of Technology, ISBN: 978-90-9025048-9

[9] R. Rubio, J. Santander, L. Fonseca, N. Sabate, I. Gracia, C. Cane, S. Udina, S. Marco, Non-selective NDIR array for gas detection, Sensors and Actuators B: Chemical, Volume 127 (2007), Issue 1, Special Issue: Eurosensors XX, Pages 69-73

[10] A Emadi, S. Grabarnik, H. Wu, G. de Graaf and R. F. Wolffenbuttel. Fabrication and characterization of infra-red multi-layered interference filter; Proceedings of MME 2007 (2009) (pp. 249-252). Guimaraes,Portugal

[11]H. Wu, S. Grabarnik, A. Emadi, G. de Graaf and R. F. Wolffenbuttel, A thermopile detector array with scaled TE elements for use in an integrated IR microspectrometer, J. Micromech. Microeng. 18064017

[12] TFCalc 3.3 (2001), Software Spectra Inc. (http://www.sspectra.com/).

[13] M.A. Rob, "Limitation of a wedged étalon for high-resolution linewidth measurements", Optics Letters, Vol. 15, Issue 11, pp. 604-606

[14] A Emadi, H. Wu, S. Grabarnik, G. de Graaf and R. F. Wolffenbuttel. Vertically tapered layers for optical applications fabricated using resist reflow; J. Micromech. Microeng. 19 (2009) 074014 (9pp)

[15] K. Mohamed, M.M. Alkaisi, Three-dimensional pattern transfer on quartz substrates, Microelectronic Engineering, Volume 87, Issues 5-8, The 35th International Conference on Micro- and Nano-Engineering (MNE), May-August 2010, Pages 1463-1466 